\title{
HOMOTOPY CLASSIFICATION OF GERBES
}

\author{
J. F. JARDine
}

Abstract

Gerbes are locally connected presheaves of groupoids on a small Grothendieck site $\mathcal{C}$. They are classified up to local weak equivalence by path components of a cocycle category taking values in the big 2-groupoid $\mathbf{I s o}(\operatorname{Gr}(\mathcal{C}))$ consisting of all sheaves of groups on $\mathcal{C}$, their isomorphisms and homotopies. If $\mathcal{F}$ is a full subpresheaf of $\operatorname{Iso}(\operatorname{Gr}(\mathcal{C}))$ then the set $[*, B \mathcal{F}]$ of morphisms in the homotopy category of simplicial presheaves classifies gerbes locally weakly equivalent to objects of $\mathcal{F}$. If $\operatorname{St}(\pi \mathcal{F})$ is the stack completion of the fundamental groupoid $\pi \mathcal{F}$ of $\mathcal{F}$, if $L$ is a global section of $\operatorname{St}(\pi \mathcal{F})$, and if $F_{L}$ is the homotopy fibre over $L$ of the canonical map $B \mathcal{F} \rightarrow B \operatorname{St}(\pi \mathcal{F})$, then $\left[*, F_{L}\right]$ is in bijective correspondence with Giraud's non-abelian cohomology object $H^{2}(\mathcal{C}, L)$ of equivalence classes of gerbes with band $L$.

\section{Contents}

Introduction

$\begin{array}{ll}\text { 1. Simplicial groupoids } & 87\end{array}$

2. The Grothendieck construction 94

3. Cocycle classification of gerbes $\quad 99$

4. Homotopy classification of gerbes 103

References 110

\section{Introduction}

Suppose that $\mathcal{M}$ is a closed model category, and that $X$ and $Y$ are objects of $\mathcal{M}$. A cocycle from $X$ to $Y$ is a picture

$$
X \stackrel{f}{\longleftarrow} Z \stackrel{g}{\rightarrow} Y
$$

2000 Mathematics Subject Classification. Primary: 20G10; Secondary: 18G30, $03 \mathrm{C} 20$.

Key words. Gerbes, cocycles, 2-groupoids, simplicial presheaves. 
of morphisms in $\mathcal{M}$ such that $f$ is a weak equivalence. A morphism of cocycles $(f, g) \rightarrow\left(f^{\prime}, g^{\prime}\right)$ is a commutative diagram<smiles>[Y][Y]12[Y]C1[I]2</smiles>

and these cocycles and their morphisms together form the category $h(X, Y)$ of cocycles from $X$ to $Y$. The assignment $(f, g) \mapsto g f^{-1}$ defines a function

$$
\phi: \pi_{0} h(X, Y) \rightarrow[X, Y]
$$

from the path components of cocycle category $h(X, Y)$ to the set of morphisms $[X, Y]$ from $X$ to $Y$ in the homotopy category $\operatorname{Ho}(\mathcal{M})$. Then it is a basic result of $[\mathbf{7}]$ that this function $\phi$ is a bijection if the model category $\mathcal{M}$ is right proper and if its class of weak equivalences is closed under finite products.

The right properness condition is a serious restriction, but right proper model structures are fairly common in nature, and include the standard model structures for spaces, simplicial sets, and spectra, as well as more exotic structures such as simplicial presheaves, simplicial sheaves and presheaves of spectra on small Grothendieck sites.

The cocycle approach to constructing morphisms in the homotopy category is proving to be very useful, particularly in connection with simplicial sheaves and presheaves. The applications so far include new, short and conceptual arguments for the homotopy classification of sheaf cohomology theories, both abelian and non-abelian [7]. Cocycle categories are involved in the explicit construction of the stack completion functor which is given in $[\mathbf{1 0}]$. They have been used to show $[\mathbf{8}]$, in a variety of settings, that morphisms $[*, B I]$ in the homotopy category can be identified with path components of a suitably defined category of $I$-torsors for small category objects $I$.

The present paper uses cocycles in presheaves of 2-groupoids, here called 2-cocycles, to give a homotopy classification of gerbes.

A gerbe is typically defined in the literature (eg. [2, p. 129]) to be a stack $G$ which is locally path connected. Stacks have no conceptual mystery - they are fibrant objects in local model structures for sheaves of groupoids [12] or more generally presheaves of groupoids [4], and this allows one to identify a stack with the local homotopy type of presheaves of groupoids that it represents. 
The model structure for presheaves (or sheaves) of groupoids, over any small Grothendieck site $\mathcal{C}$, is easy to describe. A map $f: G \rightarrow$ $H$ of presheaves of groupoids is a local weak equivalence (respectively injective fibration) if the induced map $B G \rightarrow B H$ of classifying objects is a local weak equivalence (respectively injective fibration) of simplicial presheaves. In these terms, a gerbe is a presheaf of groupoids $G$ such that the classifying simplicial presheaf $B G$ is locally path connected.

The local path connnectedness condition can be expressed this way: given objects $x, y \in \mathrm{Ob}(G)(U)$ in a section $G(U)$, there is a covering family $\phi: V \rightarrow U$ such that there is a morphism $\phi^{*}(x) \rightarrow \phi^{*}(y)$ in $G(V)$ for any $\phi$ in the cover.

Every gerbe $G$ is locally equivalent to any of its sheaves of automorphism groups. The category $h(*, \mathbf{I s o}(\operatorname{Gr}(\mathcal{C})))$ of 2-cocycles, taking values in the diagram of all sheaves of groups, their isomorphisms and homotopies, is the vehicle by which we classify gerbes up to local weak equivalence. Theorem 20 of this paper says that the path components of this cocycle category are in one to one correspondence with the path components of the category $\operatorname{Ger}(\mathcal{C})$ of gerbes and their local weak equivalences, or that there is a bijection

$$
\pi_{0}(\mathbf{G e r}(\mathcal{C})) \cong \pi_{0} h(*, \mathbf{I} \mathbf{s o}(\operatorname{Gr}(\mathcal{C}))) .
$$

One has to interpret a statement like this carefully, because the categories involved are not small. The path component functor $\pi_{0}$ means the class of equivalence classes of objects, where two objects are equivalent if and only if there is a finite string of arrows connecting them in the ambient category. We show that there are functions

$$
\Phi: \pi_{0}(\operatorname{Ger}(\mathcal{C})) \leftrightarrows \pi_{0} h(*, \mathbf{I s o}(\operatorname{Gr}(\mathcal{C}))): \Psi
$$

which are inverse to each other. Here, $\Phi$ is induced by a canonical cocycle construction which is introduced in Example 13, and $\Psi$ is defined by a generalized Grothendieck construction, which is discussed in Section 2.

The big 2-groupoid $\operatorname{Iso}(\operatorname{Gr}(\mathcal{C}))$ has subobjects which are honest presheaves of 2-groupoids. Examples include the sheaf of 2-groupoids $\operatorname{Aut}(G)$ which is associated to a sheaf of groups of $G$ on $\mathcal{C}$ : it has one object, the sheaf of 1-cells is the sheaf of automorphisms of $G$, and its sheaf of 2-cells is the sheaf of homotopies (or conjugations) of automorphisms. More generally, any presheaf of sheaves of groups in $\operatorname{Iso}(\operatorname{Gr}(\mathcal{C}))$ determines a full subobject $\mathcal{F} \subset \operatorname{Iso}(\operatorname{Gr}(\mathcal{C}))$ which is a presheaf of 2 -groupoids, and one can discuss the homotopy type of $\mathcal{F}$ and its classifying object $B \mathcal{F}$ in simplicial presheaves. It is shown in Theorem 23 that there is a one 
to one correspondences

$$
\pi_{0} h(*, \mathcal{F}) \cong \pi_{0}\left(\operatorname{Ger}_{\mathcal{F}}(\mathcal{C})\right)
$$

between the set of path components of 2-cocycles taking values in the presheaf of 2-groupoids $\mathcal{F}$ and path components of the category $\operatorname{Ger}_{\mathcal{F}}(\mathcal{C})$ of gerbes locally equivalent to sheaves of groups appearing in $\mathcal{F}$. By the result relating path components of cocycle categories to morphisms in the homotopy category displayed above, both of these objects are then in bijective correspondence with the set $[*, B \mathcal{F}]$ of morphisms in the homotopy category of simplicial presheaves - this statement appears formally as Corollary 24. The bijection of path components in the statement of Theorem 23 is a restriction of the bijection of Theorem 20.

In the special case where $\mathcal{F}=\operatorname{Aut}(G)$ for some sheaf of groups $G$, Theorem 23 says that gerbes locally equivalent to $G$ are classified up to weak equivalence by morphisms $[*, B \operatorname{Aut}(G)]$ in the homotopy category of simplicial presheaves. This result was originally proved, in a very different form, by Breen [1].

Finally, the presheaf of 2-groupoids $\mathcal{F}$ has a fundamental groupoid $\pi \mathcal{F}$ and a canonical morphism $\mathcal{F} \rightarrow \pi \mathcal{F}$. In the case where $\mathcal{F}=\operatorname{Aut}(G)$, the fundamental groupoid $\pi \operatorname{Aut}(G)$ is the sheaf of outer automorphisms of $G$. The fundamental groupoid $\pi \mathcal{F}$ has a functorial stack completion $\pi \mathcal{F} \rightarrow \operatorname{St}(\pi \mathcal{F})$, and $\operatorname{St}(\pi \mathcal{F})$ is the stack of bands (liens) for $\mathcal{F}$. Suppose that the band $L$ is a fixed choice of global section of $\operatorname{St}(\pi \mathcal{F})$, and consider the homotopy fibre $F_{L}$ of the composite

$$
B \mathcal{F} \rightarrow B \pi \mathcal{F} \rightarrow B \operatorname{St}(\pi \mathcal{F}) .
$$

Theorem 27 (see also Corollary 28) identifies the set of morphisms $\left[*, B F_{L}\right]$ in the homotopy category with path components in a suitably defined category of $L$-gerbes. In other words, Giraud's non-abelian invariant $H^{2}(\mathcal{C}, L)$ is isomorphic to $\left[*, B F_{L}\right]$. Once again, the idea of the proof is to identify the set of path components of the cocycle category $h\left(*, F_{L}\right)$ with path components in $L$-gerbes, and then use the general result about cocycles to conclude that there is a bijection

$$
\left[*, F_{L}\right] \cong \pi_{0} h\left(*, F_{L}\right) \text {. }
$$

Theorem 20, Theorem 23 and Theorem 27 are the main results of this paper. The demonstrations of these theorems appear in Sections 3 and 4 . They depend on some general statements about groupoids enriched in simplicial sets and presheaves of 2 -groupoids which are given in Section 1 , 
as well as the discussion of the generalized Grothendieck construction of Section 2 .

I would like to thank the referee for some helpful suggestions, and in particular for the observation that the Grothendieck construction is in fact a 2-category.

\section{Simplicial groupoids}

There are various equivalent ways to define a groupoid $H$ enriched in simplicial sets. The easiest thing, perhaps, is to say that such an object is a simplicial groupoid such that the simplicial set $\mathrm{Ob}(H)$ of objects is simplicially discrete, or just a set. The morphisms $\operatorname{Mor}(H)$ form a simplicial set, and the source, target $s, t: \operatorname{Mor}(H) \rightarrow \mathrm{Ob}(H)$ and identity maps $e: \mathrm{Ob}(H) \rightarrow \operatorname{Mor}(H)$ are all simplicial set maps. The notation $H_{n}$ will refer to the associated groupoid in simplicial degree $n$.

For objects $x, y$ of $H$ the simplicial set of morphisms $H(x, y)$ can be defined by the pullback diagram

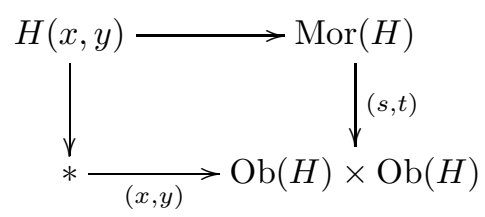

where $*=\Delta^{0}$ defines the one-point (terminal) simplicial set.

A 2-groupoid $G$ is a groupoid enriched in groupoids. Equivalently, $G$ is a groupoid enriched in simplicial sets such that the simplicial set $\operatorname{Mor}(G)$ is the nerve of a groupoid. More generally, a 2-category $A$ is a category enriched in categories, or equivalently a category enriched in simplicial sets such that the simplicial set $\operatorname{Mor}(A)$ is the nerve of a category.

One routinely writes $B H$ for both the bisimplicial set $n \mapsto B\left(H_{n}\right)$ associated to a groupoid (or category) $H$ enriched in simplicial sets and its associated diagonal simplicial set $d B H$. The vertical simplicial presheaf $B H_{n}$ in horizontal degree $n$ is the iterated fibre product defined by the inverse limit for the diagram

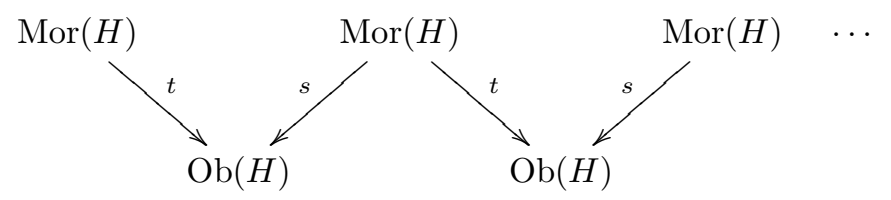

involving $n$ copies of the morphism object $\operatorname{Mor}(H)$. 
A map $G \rightarrow H$ of groupoids enriched in simplicial sets, or of 2groupoids, is said to be a weak equivalence if the induced map $d B G \rightarrow$ $d B H$ is a weak equivalence of simplicial sets.

The vertices of $B H$ are the objects of $H$, and two vertices of $B H$ are in the same path component if and only if they are in the same path component of the space $B H_{0}$, or in the same path component of the groupoid $H_{0}$ in simplicial degree 0 . It is well known and easily seen that the degeneracy morphism $H_{0} \rightarrow H_{n}$ induces a bijection $\pi_{0} B H_{0} \cong$ $\pi_{0} B H_{n}$ for all $n \geq 0$. It follows that there are natural isomorphisms

$$
\pi_{0} B H \cong \pi_{0} B H_{0} \cong \pi_{0} B H_{n}
$$

for all $n \geq 0$. I say that the object $H$ is connected if $B H$ is a pathconnected simplicial set. More generally, I write

$$
\pi_{0} H:=\pi_{0} B H,
$$

for the set of path components of $H$.

The enriched version of Quillen's Theorem B (or rather, its proof), implies that there is a homotopy cartesian diagram

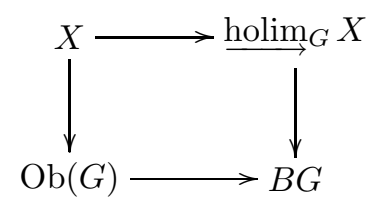

for all simplicial set-valued diagrams $X$ defined on groupoids $G$ enriched in simplicial sets [8, Lemma 2], [15].

The homotopy colimit which is displayed here and throughout this paper is the standard construction: if $X: I \rightarrow s$ Set is a small diagram of simplicial sets, then $\operatorname{holim}_{I} X$ is the bisimplicial set (or its diagonal) which is specified in vertical degree $n$ by the nerve $B\left(E_{I} X_{n}\right)$ of the translation category $E_{I} X_{n}$ which is associated to the set-valued functor $X_{n}$ - see [3, IV.1.8].

Lemma 1. Suppose that $f: G \rightarrow H$ is a morphism of groupoids enriched in simplicial sets, and that $X: H \rightarrow s$ Set is a simplicial diagram. Then the induced diagram

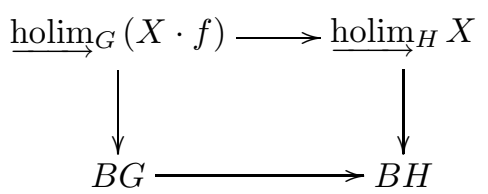

is homotopy cartesian. 
Proof: Suppose given a factorization

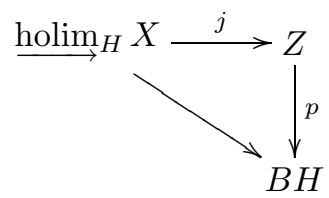

of the canonical map $\underline{\operatorname{holim}}_{H} X \rightarrow B H$ such that $j$ is a weak equivalence and $p$ is a fibration. The squares in the picture

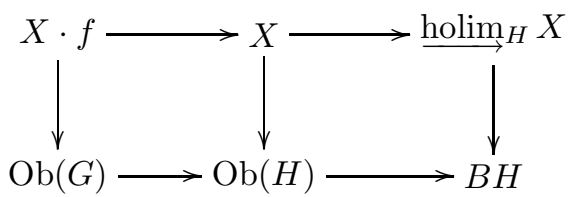

are homotopy cartesian, so that the induced map

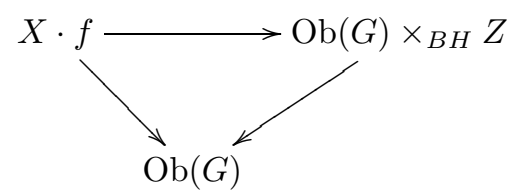

is a weak equivalence of objects over $\mathrm{Ob}(G)$. It follows that the induced map

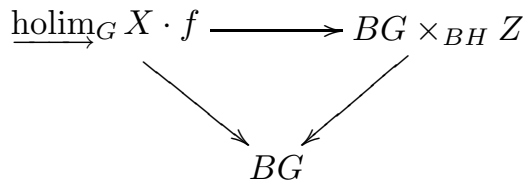

induces a weak equivalence on all homotopy fibres. This map is also a homotopy colimit of a comparison of diagrams made up of the homotopy fibres of the respective maps, and is therefore a weak equivalence.

Corollary 2. Suppose that $H$ is a groupoid enriched in simplicial sets. Then the pullback square

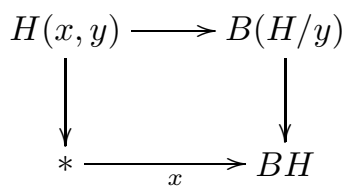

is homotopy cartesian. 
Proof: The bisimplicial set $B(H / y)$ is the homotopy colimit of a simplicial functor $H \rightarrow s$ Set which takes an object $x$ to the simplicial set $H(x, y)$, and the object $x$ defines a functor $* \rightarrow H$.

There is a simplicial groupoid $H^{\mathbf{1}}$ associated to $H$ whose objects in simplicial degree $n$ are the morphisms $h: x \rightarrow y$ of $H_{n}$ and whose morphisms $h \rightarrow h^{\prime}$ are the commutative squares

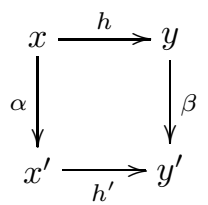

in $H_{n}$. There is a simplicial groupoid functor $(s, t): H^{\mathbf{1}} \rightarrow H \times H$ which is defined in degree $n$ by sending the square diagram above to the pair of morphisms $\left(x \stackrel{\alpha}{\rightarrow} x^{\prime}, y \stackrel{\beta}{\rightarrow} y^{\prime}\right)$. The two projections $s, t: H^{\mathbf{1}} \rightarrow H$ are weak equivalences, because they are weak equivalences in each simplicial degree.

Corollary 3. Suppose that $H$ is a groupoid enriched in simplicial sets. Then the pullback diagram

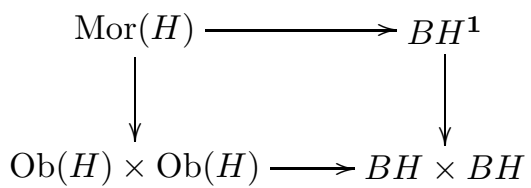

is homotopy cartesian.

Proof: The object $B H^{\mathbf{1}}$ is the homotopy colimit of a simplicial functor $\operatorname{hom}_{H}: H \times H \rightarrow s$ Set

which takes an object $(x, y)$ to the simplicial set $H(x, y)$, and which, in each simplicial degree, sends the morphism $(\alpha, \beta):(x, y) \rightarrow\left(x^{\prime}, y^{\prime}\right)$ to the function $H_{n}(x, y) \rightarrow H_{n}\left(x^{\prime}, y^{\prime}\right)$ which sends a morphism $f: x \rightarrow y$ to the composite

$$
x^{\prime} \stackrel{\alpha^{-1}}{\longrightarrow} x \stackrel{f}{\rightarrow} y \stackrel{\beta}{\rightarrow} y^{\prime} .
$$

The map $\mathrm{Ob}(H) \times \mathrm{Ob}(H) \rightarrow B H \times B H$ is induced by the inclusion of objects functor $\mathrm{Ob}(H) \times \mathrm{Ob}(H) \rightarrow H \times H$.

Let $\pi H$ denote the groupoid of path components of a groupoid $H$ enriched in simplicial sets. The object $\pi H$ will typically be called the path component groupoid of $H$. It has the same objects as $H$, and the set 
of morphisms from $x$ to $y$ is the set $\pi H(x, y)$ of path components of the simplicial set $H(x, y)$. There is a canonical map $\eta: H \rightarrow \pi H$ which is the identity on objects and is the canonical map $\operatorname{Mor}(H) \rightarrow \pi_{0} \operatorname{Mor}(H)$ on morphisms. The morphism $\eta$ is one of the canonical maps for an adjunction: the functor $H \mapsto \pi H$ is left adjoint to the inclusion of groupoids in groupoids enriched in simplicial sets.

Corollary 4. The induced map $\eta: B H \rightarrow B \pi H$ induces an isomorphism on path components and all fundamental groups, so that $\pi H$ is naturally weakly equivalent to the fundamental groupoid of $B H$.

Proof: The morphisms $H(x, x) \rightarrow \pi H(x, x)$ induce isomorphisms in path components, and so the map $B H \rightarrow B \pi H$ induces isomorphisms in path components of all loop spaces, by Corollary 2. It follows that all homomorphisms $\pi_{1}(B H, x) \rightarrow \pi_{1}(B \pi H, x)$ are isomorphisms. The claim that $\pi_{0} B H \rightarrow \pi_{0} B \pi H$ is a bijection follows from the isomorphisms of (1).

Suppose now that $\mathcal{C}$ is a small Grothendieck site. Local homotopy theory, as it is presently constituted, is based on a homotopy theory of simplicial sheaves or presheaves on such a site $\mathcal{C}$ for which the weak equivalences are defined locally [5], [11]. In particular, a map $X \rightarrow Y$ of simplicial presheaves is said to be a local weak equivalence if it induces an isomorphism $\tilde{\pi}_{0} X \cong \tilde{\pi}_{0} Y$ of sheaves of path components, and if induces isomorphisms in all possible sheaves of higher homotopy groups (with local choices of base points) in the sense that all comparisons

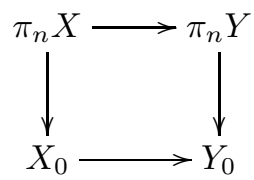

induce pullback diagrams of associated sheaves. In the presence of enough points for the sheaf category on $\mathcal{C}$, the map $f: X \rightarrow Y$ is a local weak equivalence if it induces weak equivalences $f_{x}: X_{x} \rightarrow Y_{x}$ in all stalks. A cofibration for the theory (or rather for the injective model structure on simplicial presheaves) is a monomorphism, and an injective fibration is defined by a right lifting property with respect to all maps which are cofibrations and local weak equivalences.

The injective model structure for simplicial presheaves induces a model structure on the category of presheaves of groupoids [4], for which a map $f: G \rightarrow H$ is a weak equivalence (respectively fibration) if the induced map $B G \rightarrow B H$ is a local weak equivalence (respectively injective 
fibration), and then the cofibrations are defined by a lifting property. From this point of view, a stack is, alternatively, a presheaf of groupoids which satisfies descent (ie. is sectionwise equivalent to some, hence any fibrant model), or just a homotopy type of presheaves of groupoids.

These ideas can be promoted to presheaves of higher groupoids [14]. In particular, a map $f: H \rightarrow H^{\prime}$ of presheaves of 2-groupoids is a weak equivalence if the induced map $f: d B H \rightarrow d B H^{\prime}$ is a local weak equivalence of simplicial presheaves.

If $G$ is a presheaf of groupoids on $\mathcal{C}$ and $x, y$ are objects of $G(U)$, there is a presheaf $G(x, y)$ of homomorphisms from $x$ to $y$ on $\mathcal{C} / U$. Write $G_{x}=G(x, x)$ for the presheaf of automorphisms of $x$ in $G$, and let $\tilde{G}_{x}$ denote the associated sheaf of automorphisms on $\mathcal{C} / U$.

Say that a presheaf of groupoids $G$ is a Čech object if the canonical map $G \rightarrow \pi_{0} G$ is a local weak equivalence, where $\pi_{0} G=\pi_{0} B G$ is the presheaf of path components of $G$.

In particular, an ordinary groupoid $H$ is a Cech groupoid if the groupoid morphism $H \rightarrow \pi_{0} H$ is a weak equivalence. Equivalently, $H$ is a Čech groupoid if and only if there is at most one morphism between any two objects of $H$.

Example 5. The Čech groupoid $C(p)$ for a function $p: X \rightarrow Y$ has the objects $\mathrm{Ob}(C(p))=X$, and there is a morphism $x \rightarrow y$ in $C(p)$ if and only if $p(x)=p(y)$ in $Y$. There is a canonical bijection $\pi_{0} C(p) \cong p(X)$.

This construction is natural, and therefore applies to morphisms $p: X \rightarrow Y$ of presheaves on a site. If $p$ is an epimorphism of sheaves, the simplicial presheaf map $B C(p) \rightarrow Y$ is the Cech resolution of $Y$ corresponding to the epimorphism $p$, and is a local weak equivalence. This construction produces the standard Čech resolution when applied to an epimorphism $p: \bigsqcup_{\alpha} U_{\alpha} \rightarrow Y$ arising from a covering.

Lemma 6. Suppose that $H$ is a presheaf of 2-groupoids, and let $\eta: H \rightarrow$ $\pi H$ be the canonical map to the presheaf of path component groupoids. Then $\eta$ is a local weak equivalence if and only if all presheaves of groupoids $H(x, y)$ are Čech objects.

Proof: If the map $\eta: H \rightarrow \pi H$ is a local weak equivalence, then the map

$$
\operatorname{Mor}(H) \rightarrow \operatorname{Mor}(\pi H)
$$

is a local weak equivalence over $\mathrm{Ob}(H) \times \mathrm{Ob}(H)$, by Corollary 3 . 
In general, a map

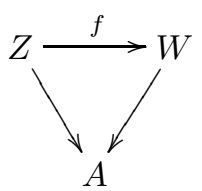

of simplicial presheaves fibred over a presheaf $A$ is a local weak equivalence if and only if it induces local weak equivalences $Z_{x} \rightarrow W_{x}$ of simplicial presheaves on $\mathcal{C} / U$ for all $x \in A(U), U \in \mathcal{C}$. Thus, if all morphism groupoids $H(x, y)$ are Čech objects, then the map $\operatorname{Mor}(H) \rightarrow$ $\operatorname{Mor}(\pi H)$ is a local weak equivalence of simplicial presheaves over the presheaf $\mathrm{Ob}(H) \times \mathrm{Ob}(H)$. It also follows that all maps

$$
\begin{aligned}
\operatorname{Mor}(H) \times_{t, s} \operatorname{Mor}(H) \times_{t, s} \cdots \times_{t, s} \operatorname{Mor}(H) \\
\rightarrow \operatorname{Mor}(\pi H) \times_{t, s} \operatorname{Mor}(\pi H) \times_{t, s} \cdots \times_{t, s} \operatorname{Mor}(\pi H)
\end{aligned}
$$

of iterated fibre products over $\mathrm{Ob}(H)$ are local weak equivalences. These are the comparison maps of vertical simplicial presheaves making up the comparison $\mathrm{BH} \rightarrow \mathrm{B \pi H}$ of bisimplicial presheaves, and one concludes that this map is a local weak equivalence.

Lemma 7. A presheaf of groupoids $H$ is a $\check{C}$ ech object if and only if for every two morphisms $f, g: x \rightarrow y$ in $H(U)$ there is a covering sieve $R \subset$ $\operatorname{hom}(, U)$ such that $\phi^{*} f=\phi^{*} g$ for all $\phi: V \rightarrow U$ in $R$.

Proof: Suppose that $H \rightarrow \tilde{H}$ is the canonical map taking values in the associated sheaf of groupoids $\tilde{H}$. Then $H$ is a Cech object if and only if all sheaves $\tilde{H}(x, x)$ of automorphisms of $\tilde{H}$ are trivial in the sense that the canonical sheaf map $\tilde{H}(x, x) \rightarrow *$ are isomorphisms. This is equivalent to the assertion that all presheaf maps $H(x, x) \rightarrow *$ are local monomorphisms.

Thus, suppose that $H$ is a Cech object, and suppose given $f, g: x \rightarrow y$ in $H(U)$ the composite $g^{-1} f \in H(x, x)(U)$, and there is a covering sieve $R \subset \operatorname{hom}(, U)$ such that $\phi^{*}\left(g^{-1} f\right)=1_{\phi^{*} x}$ for all $\phi: V \rightarrow U$ in $R$. But then $\phi^{*}(g)=\phi^{*}(f)$ for all $\phi \in R$.

The converse is clear: the local coincidence of all $f, g: x \rightarrow y$ means that all presheaf maps $H(x, y) \rightarrow *$ are local monomorphisms, and so all sheaf maps $\tilde{H}(x, x) \rightarrow *$ are isomorphisms.

Lemma 8. Suppose that $A$ is a presheaf of 2-groupoids, and that $\pi_{0} A$ is its presheaf of path components. Then the canonical map $A \rightarrow \pi_{0} A$ is a local weak equivalence if and only if all presheaves of groupoids $A(x, y)$ and the path component groupoid $\pi A$ are $\check{C}$ ech objects. 
Proof: Suppose that $A \rightarrow \pi_{0} A$ is a local weak equivalence. Then all sheaves of homotopy groups for $B A$ are trivial, and so Corollary 3 implies that all maps $A(x, y) \rightarrow *$ are local weak equivalences. In particular, all $A(x, y)$ are Cech objects. But then $\eta: A \rightarrow \pi A$ is a local weak equivalence by Lemma 6 , and so the induced map $\pi A \rightarrow \pi_{0}(\pi A)$ is a weak equivalence, so that the presheaf of groupoids $\pi A$ is a Čech object.

Suppose conversely that all $A(x, y)$ and $\pi A$ are Čech objects. Lemma 6 implies that $A \rightarrow \pi A$ is a local weak equivalence, and then the map

$$
\pi A \rightarrow \pi_{0}(\pi A) \cong \pi_{0} A
$$

is a local weak equivalence. It follows that the map $A \rightarrow \pi_{0} A$ is a composite of local weak equivalences.

\section{The Grothendieck construction}

Let cat $_{2}$ denote the 2-category whose 0 -cells are the small categories, whose 1-cells are the functors between small categories, and whose 2-cells are the homotopies of functors.

Suppose that $A$ is a 2-category, and suppose given a 2-category morphism $F: A \rightarrow$ cat $_{2}$. There is an associated 2-category $\mathbf{E}_{A}(F)$ whose 0 -cells are the pairs $(x, i)$ with $x \in F(i)$. A 1-cell $(f, \alpha):(x, i) \rightarrow(y, j)$ is a pair consisting of a 1-cell $\alpha: i \rightarrow j$ of $I$ and a morphism $f: \alpha_{*}(x) \rightarrow y$ of the category $F(j)$. Finally, a 2-cell $h:(f, \alpha) \rightarrow\left(f, \alpha^{\prime}\right)$ is a 2-cell $h: \alpha \rightarrow \alpha^{\prime}$ of $A$ such that the diagram

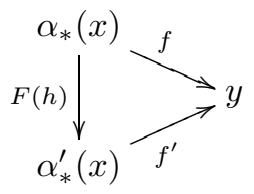

commutes, where $F(h)$ is the homotopy associated to the 2 -cell $h$ by $F$. Vertical composition of 2-cells is obvious, and horizontal composition of 2-cells is defined by the commutative diagram

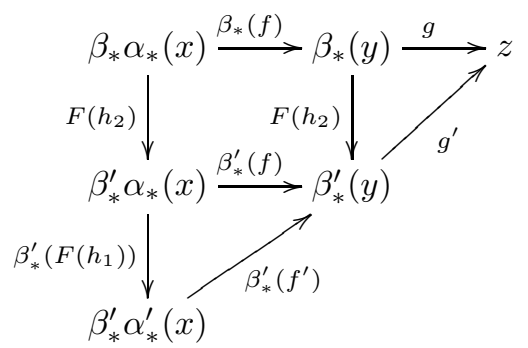


Observe that the composite homotopy $\beta_{*}^{\prime}\left(F\left(h_{1}\right)\right)\left(F\left(h_{2}\right) \alpha_{*}\right)$ is the image of the composite 2-cell $h_{2} * h_{1}$ under the morphism $F$.

There is a canonical functor of 2-categories

$$
p: \mathbf{E}_{A}(F) \rightarrow A,
$$

which is defined by the assignments $(x, i) \mapsto i$ and $(f, \alpha) \mapsto \alpha$ on 0 -cells and 1-cells, respectively, and sends the 2-cell of (2) to the 2-cell $h$ of $A$.

The path component category $\pi \mathbf{E}_{A}(F)$ of the 2-category $\mathbf{E}_{A}(F)$ is denoted by $E_{A}(F)$, and is called the Grothendieck construction for the functor $F: A \rightarrow$ cat $_{2}$. The 2-category functor $p: \mathbf{E}_{A}(F) \rightarrow A$ induces a functor

$$
p: E_{A}(F) \rightarrow \pi(A)
$$

of their associated path component categories.

Observe that if $A$ is a category enriched in groupoids, then the 1-cells $(f, \alpha),\left(f^{\prime}, \alpha^{\prime}\right):(x, i) \rightarrow(y, j)$ of $\mathbf{E}_{A}(F)$ have the same image in $E_{A}(F)$ if and only if there is a 2-cell $h: \alpha \rightarrow \alpha^{\prime}$ of $A$ such that the diagram (2) commutes.

In general, I write $[(f, \alpha)]$ for the image (path component) of the 1-cell $(f, \alpha)$ in $E_{A}(F)$.

Remark 9. Suppose that $I$ is a small category, and let $x, y$ be objects of $I$. There is a small category $I_{s}(x, y)$ whose objects are the functors $\theta: \mathbf{n} \rightarrow I$ (strings of length $n$ ) such that $\theta(0)=x$ and $\theta(n)=y$. A morphism $\theta \rightarrow \gamma$ of $I_{s}(x, y)$ is a commutative diagram of functors

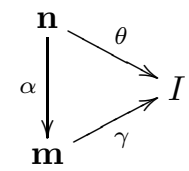

such that the ordinal number map $\alpha$ is end-point preserving in the sense that $\alpha(0)=0$ and $\alpha(n)=m$. Concatenation of strings defines a composition law $I_{s}(x, y) \times I_{s}(y, z) \rightarrow I_{s}(x, z)$, and so there is a 2-category $I_{s}$ with the same objects as $I$. There is also a canonical weak equivalence $I_{s} \rightarrow I$ (see also [3, IX.3.2]). Write $G I_{s}$ for the category enriched in groupoids, having the same 0 -cells as $I$, and such that the groupoid $G I_{s}(x, y)$ is the free groupoid on the category $I_{s}(x, y)$. The groupoid $G I_{s}(x, y)$ is a Čech groupoid with path components given by the set $I(x, y)$ of morphisms from $x$ to $y$ in $I$.

A pseudo-functor $F$ defined on $I$ and taking values in small categories can be identified with a 2-category morphism $F: G I_{s} \rightarrow$ cat $_{2}[\mathbf{3}$, IX.3.3], and one can show that the Grothendieck construction $E_{G I_{s}}(F)$ as defined 
above is isomorphic to the standard Grothendieck construction for the pseudo-functor $F$.

A lax functor $G$ defined on $I$ is a 2-category functor $G: I_{s} \rightarrow \mathbf{c a t}_{2}$. The category $E_{I_{s}}(G)$ is a Grothendieck construction for the lax functor $G$.

We shall henceforth specialize to 2-category morphisms $F: A \rightarrow \mathbf{c a t}_{2}$ which are defined on small 2-groupoids $A$.

Lemma 10. Suppose that $F: A \rightarrow \mathbf{c a t}_{2}$ is a 2-category morphism, where $A$ is a 2-groupoid. Suppose that $[(f, \alpha)]:(x, i) \rightarrow(y, j)$ is a morphism of $E_{A}(F)$ such that $f: \alpha_{*}(x) \rightarrow y$ is an invertible morphism of $F(j)$. Then $[(f, \alpha)]$ is invertible in $E_{A}(F)$.

Proof: The inverse of $[(f, \alpha)]$ is represented by $\left[\left(\alpha_{*}^{-1}\left(f^{-1}\right), \alpha^{-1}\right)\right]$.

Corollary 11. Suppose that $A$ is a 2-groupoid. If the 2-category morphism $F: A \rightarrow$ cat $_{2}$ takes values in groupoids, then $E_{A}(F)$ is a groupoid.

A diagram

$$
B \stackrel{\alpha}{\stackrel{\alpha}{\simeq}} A \stackrel{F}{\longrightarrow} \text { cat }_{2}
$$

such that $\alpha: A \rightarrow B$ is a weak equivalence of 2 -groupoids is a 2 -cocycle taking values in small categories. A morphism of 2-cocycles is a commutative diagram of functors<smiles>CCC(F)(F)[Al]1[Te][Te][Te]1</smiles>

and the corresponding 2-cocycle category is denoted by $h\left(B, \mathbf{c a t}_{2}\right)$. There are analogous definitions for 2-cocycles and 2-cocycle categories taking values in 2-categories of groups and small groupoids. These 2-cocycle categories are typically not small.

Example 12. Write Iso(Gr) for the 2-groupoid whose objects are all groups, whose 1-cells are the isomorphisms of groups $G \rightarrow H$, and whose 2-cells are the homotopies of isomorphisms, and suppose now that there is a 2-cocycle

$$
\pi A \stackrel{\eta}{\simeq} A \stackrel{K}{\longrightarrow} \mathbf{I s o}(\mathrm{Gr})
$$

where $A$ is a 2-groupoid. Then the associated Grothendieck construction $E_{A}(K)$ can be identified with a category having as objects all $i \in$ $\mathrm{Ob}(A)$ and with morphisms consisting of equivalence classes of pairs 
$(f, \alpha): i \rightarrow j$, where $\alpha: i \rightarrow j$ is a 1-cell of $A$ and $f \in K(j)$. In this case, there is a relation $(f, \alpha) \sim(g, \beta)$ if the diagram

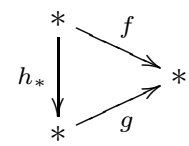

commutes in the group $K(j)$, where conjugation by $h_{*}$ defines the image of the unique 2-cell $\alpha \rightarrow \beta$. The category $E_{A}(K)$ is a groupoid by Corollary 11. It is connected if $A$ is connected, since $K$ takes values in groups.

Example 13. Suppose that $G$ is a groupoid. The resolution 2groupoid $\mathbf{R}(G)$ has the same objects and 1-cells as $G$, and has a unique 2-cell $f \rightarrow g$ between any two morphisms $f, g: x \rightarrow y$ of $G$. The path component groupoid $\pi \mathbf{R}(G)$ of $\mathbf{R}(G)$ is a Čech groupoid, and the natural maps

$$
B \mathbf{R}(G) \rightarrow B \pi \mathbf{R}(G) \rightarrow \pi_{0} B(\pi \mathbf{R}(G))
$$

are weak equivalences. There are natural bijections

$$
\pi_{0} G=\pi_{0} B G \cong \pi_{0} B(\pi \mathbf{R}(G)) .
$$

There is a canonical morphism $F(G): \mathbf{R}(G) \rightarrow \mathbf{I s o}(\mathrm{Gr})$ which takes the object $x \in G$ to the group $G_{x}=G(x, x)$, takes a 1-cell $f: x \rightarrow y$ to the isomorphism $G_{x} \rightarrow G_{y}$ which is defined by conjugation by $f$, and takes the 2-cell $f \rightarrow g$ to the homotopy defined by conjugation by the element $g f^{-1} \in G_{y}$. It follows that $G$ determines a canonical 2-cocycle

$$
\pi \mathbf{R}(G) \stackrel{\simeq}{\mathbf{R}}(G) \stackrel{F(G)}{\longrightarrow} \mathbf{I s o}(\mathrm{Gr}) .
$$

Lemma 14. There is a natural isomorphism of groupoids

$$
\psi: E_{\mathbf{R}(G)}(F(G)) \stackrel{\cong}{\longrightarrow} G
$$

which is defined fibrewise over $\pi \mathbf{R}(G)$ in the sense that there is a commutative diagram

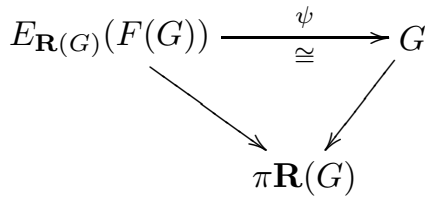

Proof: The functor $\psi$ is the identity on objects. It is defined on morphisms by sending the pair $(f, \alpha)$ to the composite $f \cdot \alpha$ in $G$. If 
$(f, \alpha) \sim(g, \beta)$ and $\alpha \rightarrow \beta$ is the unique 2 -cell in $\mathbf{R}(G)$, then the diagram

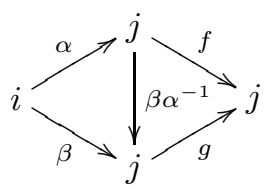

commutes in $G$, so that $f \cdot \alpha=g \cdot \beta$ and the assignment $[(f, \alpha)] \mapsto f \cdot \alpha$ is well defined. The assignment is functorial, because the diagram

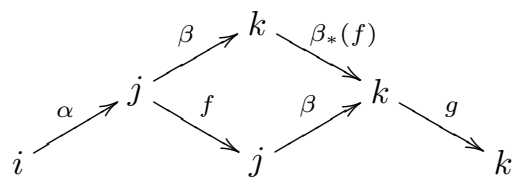

commutes in $G$.

The functor $\psi$ plainly induces surjective functions

$$
\psi: \operatorname{hom}_{E_{\mathbf{R}(G)}(F(G))}(i, j) \rightarrow \operatorname{hom}_{G}(i, j) .
$$

Finally, if the diagram

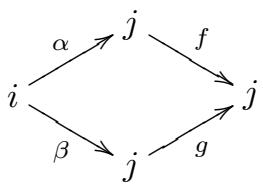

commutes in the groupoid $G$ then $g \cdot\left(\beta \alpha^{-1}\right)=f$, so that $(f, \alpha) \sim(g, \beta)$, and $\psi$ is injective on morphisms.

Remark 15. Suppose that $K: A \rightarrow \mathbf{I s o}(\mathrm{Gr})$ is a morphism of 2-groupoids, and form the resolution 2-groupoid $\mathbf{R}\left(E_{A}(K)\right)$ of the Grothendieck construction $E_{A}(K)$. Observe that the 0-cells of $\mathbf{R}\left(E_{A}(K)\right)$ coincide with the 0 -cells of $A$, the 1-cells are the morphisms $[(g, \alpha)]: i \rightarrow j$ of $E_{A}(K)$, and there is a unique 2-cell $[(g, \alpha)] \rightarrow\left[\left(g . \alpha^{\prime}\right)\right]$ for any pair of 1-cells $[(g, \alpha)],\left[\left(g^{\prime}, \alpha^{\prime}\right)\right]: i \rightarrow j$.

There is a 2-groupoid morphism $\omega: A \rightarrow \mathbf{R}\left(E_{A}(K)\right)$ which is the identity on 0-cells, sends the 1-cell $\alpha: i \rightarrow j$ to the 1 -cell $[(e, \alpha)]$ and sends a 2-cell $h: \alpha \rightarrow \beta$ to the unique 2-cell $[(e, \alpha)] \rightarrow[(e, \beta)]$.

If the map $A \rightarrow *$ is a weak equivalence then $E_{A}(K)$ is a connected groupoid and $\mathbf{R}\left(E_{A}(K)\right) \rightarrow *$ is a weak equivalence, and the map $\omega$ defines a morphism

$$
A \stackrel{\omega}{\longrightarrow} \mathbf{R}\left(E_{A}(K)\right) \stackrel{F\left(E_{A}(K)\right)}{\longrightarrow} \mathbf{I s o}(\mathrm{Gr})
$$

of 2-cocycles. 


\section{Cocycle classification of gerbes}

A gerbe $G$ is a locally connected presheaf of groupoids. A morphism of gerbes is a local weak equivalence $G \rightarrow H$ of presheaves of groupoids. We shall write $\operatorname{Ger}(\mathcal{C})$ for the category of gerbes and morphisms of gerbes on the site $\mathcal{C}$.

If $G$ is a gerbe and $x$ is a global section of $\operatorname{Ob}(G)$, then the inclusion map $G_{x} \rightarrow G$ is a local weak equivalence. It follows that every gerbe $H$ is locally equivalent to a presheaf of groups, in the sense that there is a covering $U \rightarrow *$ by objects $U \in \mathcal{C}$ and section $x_{U} \in \mathrm{Ob}(H)(U)$ such that the morphisms $\left.H_{x_{U}} \rightarrow H\right|_{U}$ are local weak equivalences over $\mathcal{C} / U$ for all $U$ in the covering.

Remark 16. Suppose that $E$ is a presheaf, and identify $E$ with a presheaf of discrete groupoids. An $E$-gerbe is a morphism $G \rightarrow E$ of presheaves of groupoids such that the associated presheaf map $\pi_{0} G \rightarrow E$ induces an isomorphism $\tilde{\pi}_{0} G \cong \tilde{E}$ of associated sheaves. A morphism of $E$-gerbes is a commutative diagram

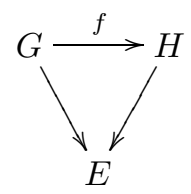

such that the morphism $f: G \rightarrow H$ is a local weak equivalence of presheaves of groupoids. Write $\mathbf{G e r}_{E}(\mathcal{C})$ for the corresponding category.

There is an equivalence of categories

$$
\operatorname{Ger}_{E}(\mathcal{C}) \simeq \operatorname{Ger}(\mathcal{C} / E)
$$

between the category of $E$-gerbes on $\mathcal{C}$ and the category of gerbes for the fibred site $\mathcal{C} / E$. Equivalences of this sort are discussed in [9]. Classification results for $E$-gerbes can therefore be deduced from classification results for gerbes on the site $\mathcal{C} / E$.

Categories of $E$-gerbes appear in applications - see [13, p. 22], for example.

We shall write $\operatorname{Iso}(\operatorname{Gr}(\mathcal{C}))$ for the following monster: it is a contravariant diagram defined on $\mathcal{C}$ and taking values in 2-groupoids, such that the 0-cells of $\operatorname{Iso}(\operatorname{Gr}(\mathcal{C}))(U)$ are the sheaves of groups on $\mathcal{C} / U$, the 1-cells are the isomorphisms of sheaves of groups on $\mathcal{C} / U$, and the 2-cells are the homotopies of sheaf isomorphisms. The object $\operatorname{Iso}(\operatorname{Gr}(\mathcal{C}))$ is not a presheaf of groupoids, because it does not take values in small groupoids. 
If $G$ is a gerbe, then the corresponding resolution 2-groupoid $\mathbf{R}(G)$ (Example 13) is weakly equivalent to a point in the sense that the map $\mathbf{R}(G) \rightarrow *$ is a local weak equivalence of presheaves of 2 -groupoids.

There is a canonical morphism

$$
F(G): \mathbf{R}(G) \rightarrow \mathbf{I s o}(\operatorname{Gr}(\mathcal{C}))
$$

for which the 0-cell $x \in \mathbf{R}(G)(U)$ is mapped to the sheaf of groups $\tilde{G}_{x}$, the 1-cell $\alpha: x \rightarrow y$ is mapped to the sheaf isomorphism $c_{\alpha}: \tilde{G}_{x} \rightarrow \tilde{G}_{y}$ on $\mathcal{C} / U$ which is defined by conjugation by the global section $\alpha$, and each 2-cell $h: \alpha \rightarrow \beta$ between 1-cells $x \rightarrow y$ maps to conjugation by the image of $h \in \tilde{G}_{y}(U)$ in global sections of $\tilde{G}_{y}$.

In this way, each gerbe $G$ has a canonically associated 2-cocycle

$$
* \stackrel{\simeq}{R}(G) \stackrel{F(G)}{\longrightarrow} \mathbf{I s o}(\operatorname{Gr}(\mathcal{C})) .
$$

Write $h(*, \mathbf{I s o}(\operatorname{Gr}(\mathcal{C})))$ for the category of 2-cocycles taking values in the 2-groupoid object $\mathbf{I s o}(\operatorname{Gr}(\mathcal{C}))$.

A map $G \rightarrow H$ of gerbes induces a 2-groupoid morphism $f_{*}: \mathbf{R}(G) \rightarrow$ $\mathbf{R}(H)$, but the assignment of the cocycle $F(G): \mathbf{R}(G) \rightarrow \operatorname{Iso}(\operatorname{Gr}(\mathcal{C}))$ to the gerbe $G$ is not quite functorial. The sheaf isomorphisms $f_{x}: \tilde{G}_{x} \cong$ $\tilde{H}_{f(x)}$ which are induced by the local weak equivalence $f$ determine a homotopy

$$
h_{f}: \mathbf{R}(G) \times \underline{\mathbf{1}} \rightarrow \mathbf{I s o}(\operatorname{Gr}(\mathcal{C}))
$$

from $F(G)$ to $F(H) \cdot f_{*}$. It follows that $F(G)$ and $F(H)$ represent the same element of $\pi_{0} h(*, \mathbf{I s o}(\operatorname{Gr}(\mathcal{C})))$, and so the assignment $G \mapsto[F(G)]$ induces a function

$$
\Phi: \pi_{0} \operatorname{Ger}(\mathcal{C}) \rightarrow \pi_{0} h(*, \mathbf{I s o}(\operatorname{Gr}(\mathcal{C}))) .
$$

Suppose that

$$
* \stackrel{\text { 에 }}{\longleftarrow} \stackrel{K}{\longrightarrow} \mathbf{I s o}(\operatorname{Gr}(\mathcal{C}))
$$

is a 2-cocycle taking values in $\operatorname{Iso}(\operatorname{Gr}(\mathcal{C}))$. Then $K$ consists of 2-groupoid morphisms $K(U): A(U) \rightarrow \mathbf{I s o}(\operatorname{Gr}(\mathcal{C}))(U)$, and hence induces composite morphisms

$$
A(U) \stackrel{K(U)}{\longrightarrow} \mathbf{I s o}(\operatorname{Gr}(\mathcal{C}))(U) \stackrel{e v_{U}}{\longrightarrow} \mathbf{I s o}(\mathrm{Gr}) .
$$

Here, $e v_{U}: \operatorname{Iso}(\operatorname{Gr}(\mathcal{C}))(U) \rightarrow \mathbf{I s o}(\mathrm{Gr})$ is the 2-groupoid morphism which is defined by $U$-sections.

Write $E_{A}(K(U))$ for the Grothendieck construction corresponding to the composite $e v_{U} K(U)$. Then the assignment $U \mapsto E_{A}(K(U))$ defines 
a presheaf of groupoids $E_{A}(K)$. From Section 2, we see that there is a canonical morphism

$$
p: E_{A}(K) \rightarrow \pi A
$$

of presheaves of groupoids; it is defined in sections to be the identity on objects, and it sends a class $[(f, \alpha)]$ to the class $[\alpha]$.

Lemma 17. Suppose that $K: A \rightarrow \operatorname{Iso}(\operatorname{Gr}(\mathcal{C}))$ is a 2-cocycle over the terminal object $*$. Then the presheaf of groupoids $E_{A}(K)$ is a gerbe.

Proof: The map $\pi_{0} E_{A}(K) \rightarrow \pi_{0}(\pi A)$ is an isomorphism of presheaves, since each 2 -functor $e v_{U} K(U)$ takes values in groups. The sheaf associated to $\pi_{0}(A) \cong \pi_{0}(\pi A)$ is trivial, since $A$ is weakly equivalent to a point as a 2-groupoid.

Lemma 18. Suppose that $K: A \rightarrow \mathbf{I s o}(\operatorname{Gr}(\mathcal{C}))$ is a 2-cocycle over $*$, and choose $i \in A(U)$. Then the homomorphism $\gamma_{i}: K(i) \rightarrow E_{A}(K)(i, i)$ which is defined by $f \mapsto\left[\left(f, 1_{i}\right)\right]$ induces an isomorphism of sheaves of groups on $\mathcal{C} / U$.

Proof: Suppose that $[(g, \alpha)]$ is an element of hom $(i, i)$. By Lemma 7 there is a covering sieve $R \subset \operatorname{hom}(, U)$ such that there is a 2-cell $h_{\phi}: \phi^{*}(\alpha) \rightarrow 1_{\alpha^{*}(i)}$ for all $\phi \in R$. It follows that, locally, $[(g, \alpha)]$ is in the image of $\gamma_{i}$.

Take group elements $f, g \in K(i)(U)$ and suppose that $\gamma_{i}(f)=\gamma_{i}(g)$. Then there is a 2-cell $h: 1_{i} \rightarrow 1_{i}$ in $A(U)$ such that the diagram

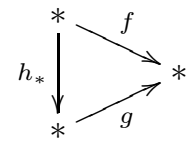

commutes in $K(i)(U)$. The presheaf of groupoids $A(i, i)$ is a Čech object by Lemma 8 so that there is a covering $\phi: V \rightarrow U$ such that $\phi^{*}(h)=1$ for all members $\phi$ of the cover. But then $\phi^{*}\left(h_{*}\right)=1$ for all $\phi$, and so $h_{*}=1$ since $K(i)$ is a sheaf of groups.

Corollary 19. Suppose that the diagram

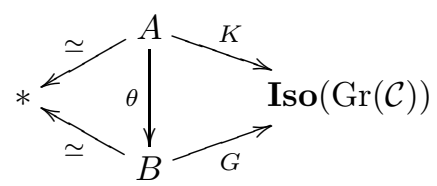

is a morphisms of 2-cocycles. Then the induced map $\theta: E_{A}(K) \rightarrow E_{B}(G)$ is a local weak equivalence of presheaves of groupoids. 
Proof: The diagram

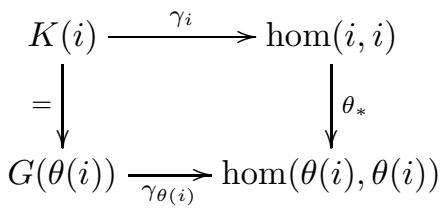

commutes, so that $\theta$ induces an isomorphism on all sheaves of fundamental groups by Lemma 18 . The objects $E_{A}(K)$ and $E_{B}(G)$ are locally path connected by Lemma 17.

It follows that the assignment $K \mapsto E_{A}(K)$ defines a functor

$$
h(*, \mathbf{I s o}(\operatorname{Gr}(\mathcal{C}))) \rightarrow \operatorname{Ger}(\mathcal{C}),
$$

and hence induces a function

$$
\Psi: \pi_{0} h(*, \mathbf{I s o}(\operatorname{Gr}(\mathcal{C}))) \rightarrow \pi_{0}(\operatorname{Ger}(\mathcal{C})) .
$$

Theorem 20. The functions $\Phi$ and $\Psi$ are inverse to each other, and define a bijection

$$
\pi_{0}(\operatorname{Ger}(\mathcal{C})) \cong \pi_{0} h(*, \mathbf{I s o}(\operatorname{Gr}(\mathcal{C})))
$$

Proof: The relation $\Psi \Phi=1$ is a consequence of Lemma 14 .

Suppose that $K: A \rightarrow \mathbf{I s o}(\operatorname{Gr}(\mathcal{C}))$ is a 2-cocycle over $*$. Following Remark 15, there is a 2-groupoid morphism $\omega: A \rightarrow \mathbf{R}\left(E_{A}(K)\right)$ which is the identity on objects, sends the 1 -cell $\alpha: i \rightarrow j$ to the 1 -cell $[(e, \alpha)]$, and sends the 2 -cell $h: \alpha \rightarrow \beta$ to the unique 2 -cell $[(e, \alpha)] \rightarrow[(e, \beta)]$. The composite

$$
A \stackrel{\omega}{\longrightarrow} \mathbf{R}\left(E_{A}(K)\right) \stackrel{F\left(E_{A}(K)\right)}{\longrightarrow} \mathbf{I s o}(\operatorname{Gr}(\mathcal{C}))
$$

defines a group-valued 2-cocycle on $\pi_{0} A$. This composite sends the object $i \in A$ to the presheaf of groups $E_{A} K(i, i)$, sends a 1-cell $\alpha: i \rightarrow j$ to the homomorphism $c_{\alpha}: E_{A} K(i, i) \rightarrow E_{A} K(j, j)$ which is defined by conjugation with $[(e, \alpha)]$, and sends a 2-cell $h: \alpha \rightarrow \beta$ to the homotopy defined by conjugation with the element

$$
\left[\left(h_{*}, 1\right)\right]=[(e, \beta)][(e, \alpha)]^{-1} .
$$

The assignments $f \mapsto[(f, 1)]$ define homomorphisms

$$
\gamma_{i}: K(i) \rightarrow E_{A} K(i, i)
$$

which induce isomorphisms of associated sheaves, by Lemma 18 . The morphisms $\gamma_{i}$ further determine a homotopy

$$
\gamma: A \times \underline{\mathbf{1}} \rightarrow \operatorname{Iso}(\operatorname{Gr}(\mathcal{C}))
$$


from the cocycle $K$ to the cocycle $F\left(E_{A}(K)\right) \omega$. It follows that there is a path

$$
F\left(E_{A}(K)\right) \sim F\left(E_{A}(K)\right) \omega \sim \gamma \sim K
$$

in the cocycle category, and so $\Psi \Phi=1$ as required.

\section{Homotopy classification of gerbes}

Suppose that $\mathcal{F} \subset \mathbf{I s o}(\operatorname{Gr}(\mathcal{C}))$ is a subobject of $\operatorname{Iso}(\operatorname{Gr}(\mathcal{C}))$ such that 1) the imbedding is full in the sense that all simplicial presheaf maps

$$
\mathcal{F}(H, K) \rightarrow \mathbf{I s o}(\operatorname{Gr}(\mathcal{C}))(H, K)
$$

are isomorphisms.

2) $\mathcal{F}$ is a presheaf of 2-groupoids, so that all classes $\operatorname{Ob}(\mathcal{F})(U)$ are sets.

We shall say that a subobject $\mathcal{F}$ of the diagram of 2-groupoids $\operatorname{Iso}(\operatorname{Gr}(\mathcal{C}))$ which satisfies these conditions is a full subpresheaf of $\operatorname{Iso}(\operatorname{Gr}(\mathcal{C}))$.

Suppose that $G$ is a gerbe, and let $\mathcal{F}_{G} \subset \operatorname{Iso}(\operatorname{Gr}(\mathcal{C}))$ be the smallest full subpresheaf which contains the image of the cocycle $F(G): \mathbf{R}(G) \rightarrow$ Iso $(\operatorname{Gr}(\mathcal{C}))$. It is the full subobject of $\operatorname{Iso}(\operatorname{Gr}(\mathcal{C}))$ whose objects are the automorphism sheaves of groups $\tilde{G}_{x}$ associated to the automorphism groups $G_{x}$ of $G$, where $x \in \operatorname{Ob}(G)(U), U \in \mathcal{C}$. The 2-cocycle $F(G): \mathbf{R}(G) \rightarrow \operatorname{Iso}(\operatorname{Gr}(\mathcal{C}))$ factors uniquely through a cocycle $\mathbf{R}(G) \rightarrow$ $\mathcal{F}_{G}$.

Lemma 21. Suppose that $\mathcal{F} \subset \mathcal{F}^{\prime}$ are full subpresheaves of $\operatorname{Iso}(\operatorname{Gr}(\mathcal{C}))$. Suppose further that every object $\mathcal{F}_{x}^{\prime}$ of $\mathcal{F}^{\prime}$ is locally isomorphic to an object of $\mathcal{F}$. Then the inclusion $\mathcal{F} \subset \mathcal{F}^{\prime}$ is a local weak equivalence of presheaves of 2-groupoids.

Proof: Write $\alpha: \mathcal{F} \subset \mathcal{F}^{\prime}$ for the inclusion morphism. Then $\alpha$ is full, and therefore induces a presheaf monomorphism $\pi_{0} \mathcal{F} \rightarrow \pi_{0} \mathcal{F}^{\prime}$. Every object of $\mathcal{F}_{x}^{\prime} \in \mathcal{F}^{\prime}(U)$ is locally isomorphic to an object in the image of $\alpha$, by assumption, so that $\pi_{0} \mathcal{F} \rightarrow \pi_{0} \mathcal{F}^{\prime}$ is a local epimorphism.

The assertion that $\alpha$ induces an isomorphism in all sheaves of higher homotopy groups is a consequence of the fullness of $\alpha$ along with Corollary 2.

Say that two gerbes $G$ and $H$ are locally equivalent if there is a covering family $U \rightarrow *, U \in \mathrm{Ob}(\mathcal{C})$, such that the restricted gerbes $\left.G\right|_{U}$ and $\left.H\right|_{U}$ are (locally) weakly equivalent on $\mathcal{C} / U$ for each object $U$ in the covering of the terminal object $*$. If there is a local weak equivalence $G \rightarrow H$ then $G$ and $H$ are locally equivalent in the sense just described, but the converse assertion is false. 
Example 22. Suppose that the presheaf of 2 -groupoids $\mathcal{F}$ is a full subpresheaf of $\operatorname{Iso}(\operatorname{Gr}(\mathcal{C}))$, and that there is a 2-cocycle

$$
* \stackrel{\simeq}{\longleftarrow} A \stackrel{F}{\rightarrow} \mathcal{F} \subset \mathbf{I s o}(\operatorname{Gr}(\mathcal{C}))
$$

over the terminal sheaf $*$.

There is a covering family $U \rightarrow *, U \in \mathcal{C}$, such that $A(U) \neq \emptyset$. In effect, $\mathrm{Ob}(A) \rightarrow *$ is a local epimorphism, so there is a covering $U \rightarrow *$ such that there are liftings

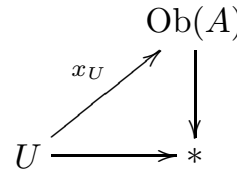

where $x_{U}$ represents an object of $A(U)$. The presheaf of groupoids $E_{A}(F)$ is locally connected by Lemma 17 , and the maps

$$
F\left(x_{U}\right) \rightarrow \operatorname{hom}_{E_{A}(F)}\left(x_{U}, x_{U}\right)
$$

induce isomorphisms of associated sheaves of groups on $\mathcal{C} / U$ by Lemma 18. It follows that the automorphism groups of the Grothendieck construction $E_{A}(F)$ are locally isomorphic to objects of $\mathcal{F}$.

Write $\operatorname{Ger}_{\mathcal{F}}(\mathcal{C})$ for the full subcategory of the category of gerbes whose automorphism groups are locally isomorphic to sheaves of groups in $\mathcal{F}$. I also say that the objects of this category are $\mathcal{F}$-gerbes. The assignment $F \mapsto E_{A}(F)$ for a cocycle $F: A \rightarrow \mathcal{F}$ takes values in $\mathcal{F}$-gerbes, so that there is a commutative diagram

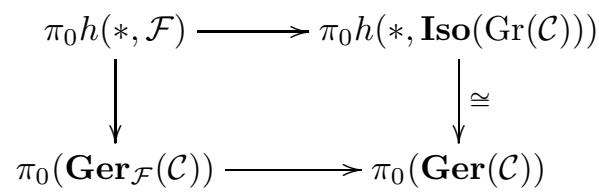

Note that if $f: G \rightarrow H$ is a local weak equivalence of gerbes, then $G$ is an $\mathcal{F}$-gerbe if and only if $H$ is an $\mathcal{F}$-gerbe, and it follows that the induced map

$$
\pi_{0}\left(\operatorname{Ger}_{\mathcal{F}}(\mathcal{C})\right) \rightarrow \pi_{0}(\operatorname{Ger}(\mathcal{C}))
$$

is an injection.

Theorem 23. Suppose that $\mathcal{F}$ is a full subpresheaf of the $\operatorname{Iso}(\operatorname{Gr}(\mathcal{C}))$. Then the Grothendieck construction defines a function

$$
\pi_{0} h(*, \mathcal{F}) \rightarrow \pi_{0}\left(\operatorname{Ger}_{\mathcal{F}}(\mathcal{C})\right)
$$

which is a bijection. 
Proof: Suppose given cocycles $F: A \rightarrow \mathcal{F}$ and $G: B \rightarrow \mathcal{F}$ such that $F$ and $G$ are in the same path component as cocycles taking values in Iso $(\operatorname{Gr}(\mathcal{C}))$. Then there is a string of maps of cocycles

$$
F=F_{0} \leftrightarrow F_{1} \leftrightarrow \cdots \leftrightarrow F_{n}=G
$$

where $F_{i}: A_{i} \rightarrow \mathbf{I s o}(\operatorname{Gr}(\mathcal{C}))$ are cocycles in $\mathbf{I s o}(\operatorname{Gr}(\mathcal{C}))$.

Suppose that $F: A \rightarrow \mathbf{I s o}(\operatorname{Gr}(\mathcal{C}))$ is a cocycle taking values in sheaves of groups locally isomorphic to objects of $\mathcal{F}$ and that

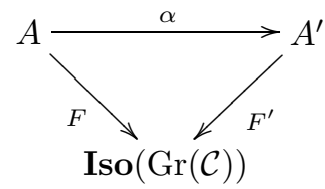

is a morphism of $h(*, \mathbf{I s o}(\operatorname{Gr}(\mathcal{C})))$. Take $x \in A^{\prime}(U)$. Then there is a covering family $\phi: V \rightarrow U$ with 1-cells $\phi^{*}(x) \rightarrow \alpha\left(y_{V}\right)$ in $A^{\prime}(V)$ for all $\phi$. It follows that the group $F^{\prime}(x)$ is locally isomorphic to groups of the form $F\left(y_{V}\right)$, and all of these are locally isomorphic to objects of $\mathcal{F}$. Thus, the cocycle $F^{\prime}$ takes values in sheaves of groups locally isomorphic to objects of $\mathcal{F}$.

It follows that all cocycles $F_{i}$ in the list (4) take values in groups locally isomorphic to objects of $\mathcal{F}$. Write $\mathcal{F}^{\prime}$ for the presheaf of 2 -groupoids which is the full subobject of $\operatorname{Iso}(\operatorname{Gr}(\mathcal{C}))$ on the sheaves of groups appearing in the sets $\mathcal{F}(U)$ and all $F_{i}\left(\mathrm{Ob}\left(A_{i}\right)\right)(U)$. Then $\mathcal{F} \subset \mathcal{F}^{\prime}$, and Lemma 21 implies that this map of presheaves of 2 -groupoids is a weak equivalence. The string of cocycles $F_{i}$ in (4) all take values in $\mathcal{F}^{\prime}$ by construction, and the map

$$
\pi_{0} h(*, \mathcal{F}) \rightarrow \pi_{0} h\left(*, \mathcal{F}^{\prime}\right)
$$

is a bijection. It follows that the original cocycles $F$ and $G$ are in the same path component of $h(*, \mathcal{F})$. The function

$$
\pi_{0} h(*, \mathcal{F}) \rightarrow \pi_{0} h(*, \mathbf{I s o}(\operatorname{Gr}(\mathcal{C})))
$$

is therefore a monomorphism, as is the function

$$
\pi_{0} h(*, \mathcal{F}) \rightarrow \pi_{0}\left(\operatorname{Ger}_{\mathcal{F}}(\mathcal{C})\right) .
$$

Suppose that $H$ is a gerbe such that every automorphism sheaf $\tilde{H}_{x}$ is locally isomorphic to an object of $\mathcal{F}$, so that $H$ is an object of $\operatorname{Ger}_{\mathcal{F}}(\mathcal{C})$. Choose a full subpresheaf $\mathcal{F}^{\prime} \subset \operatorname{Iso}(\operatorname{Gr}(\mathcal{C}))$ whose 0-cells are sheaves of groups locally equivalent to objects of $\mathcal{F}$ and which contains both $\mathcal{F}$ and $\mathcal{F}_{H}$. Then the canonical cocycle

$$
F(H): \mathbf{R}(H) \rightarrow \mathbf{I s o}(\operatorname{Gr}(\mathcal{C}))
$$


takes values in $\mathcal{F}^{\prime}$. The map $\mathcal{F} \rightarrow \mathcal{F}^{\prime}$ is a local weak equivalence, and so the class of $F(H)$ in $\pi_{0} h(*, \mathbf{I s o}(\operatorname{Gr}(\mathcal{C})))$ can be represented by a cocycle taking values in $\mathcal{F}$. It follows that the function $\pi_{0} h(*, \mathcal{F}) \rightarrow$ $\pi_{0}\left(\operatorname{Ger}_{\mathcal{F}}(\mathcal{C})\right)$ is surjective.

Corollary 24. Suppose that $\mathcal{F}$ is a full subpresheaf of 2-groupoids in the object $\operatorname{Iso}(\operatorname{Gr}(\mathcal{C}))$. Then there is a bijection

$$
[*, d B \mathcal{F}] \cong \pi_{0}\left(\operatorname{Ger}_{\mathcal{F}}(\mathcal{C})\right) \text {. }
$$

Recall that $d B \mathcal{F}$ is the diagonal of the bisimplicial set $B \mathcal{F}$ which is associated to the 2-groupoid $\mathcal{F}$.

Suppose that $G$ is a gerbe, and write $\operatorname{Ger}_{G}(\mathcal{C})$ for the category of gerbes which are locally equivalent to $G$. This category coincides with the category $\operatorname{Ger}_{\mathcal{F}_{G}}(\mathcal{C})$, and so we have the following:

Corollary 25. Suppose that $G$ is a gerbe on a site $\mathcal{C}$, with associated 2-groupoid object $\mathcal{F}_{G}$ of isomorphisms and homotopies of automorphism sheaves of $G$. Then there is a bijection

$$
\left[*, d B \mathcal{F}_{G}\right] \cong \pi_{0}\left(\operatorname{Ger}_{G}(\mathcal{C})\right) .
$$

Remark 26. A special case of Corollary 25, corresponding to the case of a sheaf of groups $G$, was proved by Breen in $[\mathbf{1}]$. To see the connection, observe that there is an isomorphism

$$
\operatorname{Aut}(G) \stackrel{\cong}{\longrightarrow} \mathcal{F}_{G}
$$

which sends the object $*$ to the object $G$, since $G$ is a one-object groupoid.

Suppose that $\mathcal{F}$ is a full subpresheaf of $\operatorname{Iso}(\operatorname{Gr}(\mathcal{C}))$, and write $\operatorname{Ger}(\mathcal{F})$ for the full subcategory of $\operatorname{Ger}(\mathcal{C})$ whose objects are the gerbes $G$ such that $\mathcal{F}_{G} \subset \mathcal{F}$. The category $\operatorname{Ger}_{\mathcal{F}}(\mathcal{C})$ is a filtered colimit of subcategories $\operatorname{Ger}\left(\mathcal{F}^{\prime}\right)$, indexed over all inclusions $\mathcal{F} \subset \mathcal{F}^{\prime}$ of full subpresheaves of $\mathbf{I s o}(\operatorname{Gr}(\mathcal{C}))$ such that every object of $\mathcal{F}^{\prime}$ is locally isomorphic to objects of $\mathcal{F}$. It follows that there is an isomorphism

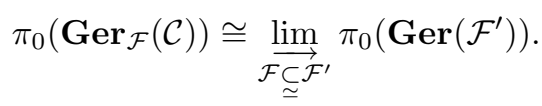

Write $\operatorname{St}(\mathcal{F})$ and $\operatorname{St}(\pi \mathcal{F})$ for the stack completions (fibrant models) for the presheaf of 2-groupoids $\mathcal{F}$ and its path component object $\pi \mathcal{F}$. The path component object is a groupoid of outer automorphisms and its stack completion $\operatorname{St}(\pi \mathcal{F})$ is the stack of bands (liens) for $\mathcal{F}$. The stack completions are functorial, since the underlying model structures are cofibrantly generated [14]. 
A band $L$ is a global section of the presheaf of groupoids $\operatorname{St}(\pi \mathcal{F})$, or equivalently [10] a torsor for the presheaf of outer automorphism groupoids $\pi \mathcal{F}$.

Write $p_{\mathcal{F}}$ for the composite

$$
\mathcal{F} \rightarrow \pi \mathcal{F} \rightarrow \operatorname{St}(\pi \mathcal{F}) .
$$

The homotopy fibre over a band $L$ of the induced map $B \mathcal{F} \rightarrow B \operatorname{St}(\pi \mathcal{F})$ is the classifying object $B\left(p_{\mathcal{F}} / L\right)$ of the simplicial groupoid $p_{\mathcal{F}} / L[\mathbf{8}]$.

The objects of 2-cocycle category $h\left(*, p_{\mathcal{F}} / L\right)$ can be identified with the collection of pairs $(\phi, \nu)$ consisting of a 2 -cocycle

$$
* \stackrel{\varrho}{\longleftarrow} \stackrel{\phi}{\rightarrow} \mathcal{F}
$$

and a natural isomorphism $\nu: \phi_{*} \rightarrow L$ in $\operatorname{St}(\pi \mathcal{F})$, where $\phi_{*}: \pi(A) \rightarrow$ $\operatorname{St}(\pi \mathcal{F})$ is the composite

$$
\pi(A) \rightarrow \pi \mathcal{F} \rightarrow \operatorname{St}(\pi \mathcal{F}) .
$$

The morphisms $f:(\phi, \nu) \rightarrow\left(\phi^{\prime}, \nu^{\prime}\right)$ are cocycle morphisms

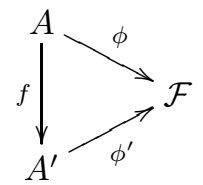

such that $\nu^{\prime} \cdot f_{*}=\nu: \phi_{*} \rightarrow L$.

Recall that a gerbe $G$ in $\mathcal{F}$ is defined by the canonical cocycle for $G$ having the form $F(G): \mathbf{R}(G) \rightarrow \mathcal{F}$.

An $L$-gerbe $(G, \mu)$ is an $\mathcal{F}$-gerbe $G$, together with a natural isomorphism $\mu: F(G)_{*} \rightarrow L$ in $\operatorname{St}(\pi \mathcal{F})$ so that $(F(G), \mu)$ defines an object of the 2-cocycle category $h\left(*, p_{\mathcal{F}} / L\right)$.

There is a canonical natural isomorphism

$$
h_{f *}: F(G)_{*} \cong F(H)_{*} f_{*}
$$

for any morphism $f: G \rightarrow H$ of gerbes which is induced by the homotopy $h_{f}$ of (3). A morphism $f:(G, \mu) \rightarrow\left(H, \mu^{\prime}\right)$ of $L$-gerbes is a morphism $f: G \rightarrow H$ of gerbes such that the diagram of natural isomorphisms

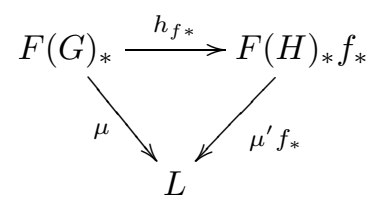


commutes. The natural isomomorphisms $h_{f *}: G(x, x) \rightarrow G(f(x), f(x))$ arising from gerbe morphisms $f$ are coherent; this gives the law of composition for a category of $L$-gerbes in $\mathcal{F}$, which will be denoted by $\operatorname{Ger}(\mathcal{F}) / L$.

Every $L$-gerbe $(G, \mu)$ in $\mathcal{F}$ determines an object $(F(G), \mu)$ in the cocycle category $h\left(*, p_{\mathcal{F}} / L\right)$, by definition.

Suppose that $f:(G, \mu) \rightarrow\left(H, \mu^{\prime}\right)$ is a morphism of $L$-gerbes in $\mathcal{F}$. Then the homotopy of cocycles $h_{f}: \mathbf{R}(G) \times \underline{\mathbf{1}} \rightarrow \mathcal{F}$ from $F(G)$ to $F(H) f_{*}$ determines a diagram morphisms

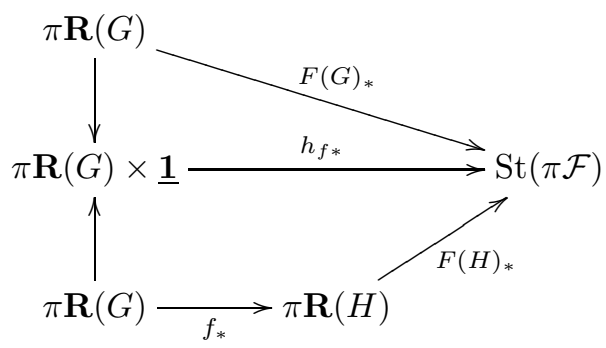

The natural isomorphism $\mu: F(G)_{*} \rightarrow L$ extends uniquely to a natural isomorphism $\mu_{h}: h_{f *} \rightarrow L$, and $\mu_{h}$ restricts to $\mu^{\prime} f_{*}: F(H)_{*} \rightarrow L$ on $\pi \mathbf{R}(G) \times\{1\}$ on account of the commutativity of the diagram (5).

It follows that every morphism $f:(G, \mu) \rightarrow\left(H, \mu^{\prime}\right)$ of $L$-gerbes determines a path between the associated objects $(F(G), \mu),\left(F(H), \mu^{\prime}\right)$ in the cocycle category, and that there is a function

$$
\Phi_{\mathcal{F}}: \pi_{0}(\operatorname{Ger}(\mathcal{F}) / L) \rightarrow \pi_{0} h\left(*, p_{\mathcal{F}} / L\right)
$$

which is defined by $\Phi([(G, \mu)])=[(F(G), \mu)]$.

Suppose that the 2-cocycle

$$
* \stackrel{\simeq}{\longleftarrow} A \stackrel{\phi}{\rightarrow} \mathcal{F}
$$

and the natural isomorphism $\nu: \phi_{*} \rightarrow L$ define an object $(\phi, \nu)$ of the cocycle category $h\left(*, p_{\mathcal{F}} / L\right)$. Then the associated presheaf of groupoids $E_{A}(\phi)$ is a gerbe which has automorphism sheaves locally isomorphic to objects of $\mathcal{F}$, and from the proof of Theorem 20 we know 
that there is a homotopy

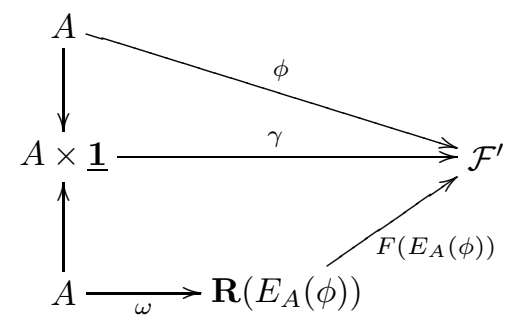

where $\mathcal{F}^{\prime}$ is a full subpresheaf of $\operatorname{Iso}(\operatorname{Gr}(\mathcal{C}))$ containing $\mathcal{F}$ such that the map $\mathcal{F} \subset \mathcal{F}^{\prime}$ is a local weak equivalence. We also know that the induced map $\omega_{*}: \pi A \rightarrow \pi \mathbf{R}\left(E_{A}(\phi)\right)$ is an isomorphism. It follows that the induced natural isomorphism (or homotopy)

$$
\gamma_{*}: \phi_{*} \cong F\left(E_{A}(\phi)\right)_{*} \omega_{*}
$$

of functors $\pi A \rightarrow \operatorname{St}\left(\mathcal{F}^{\prime}\right)$ induces a unique natural isomorphism

$$
F\left(E_{A}(\phi)\right)_{*} \stackrel{\tilde{\nu}}{\rightarrow} L
$$

which restricts to the isomorphism $\nu: \phi_{*} \rightarrow L$ along the homotopy

$$
\pi(A) \times \underline{\mathbf{1}} \stackrel{\gamma_{*}}{\longrightarrow} \operatorname{St}\left(\pi \mathcal{F}^{\prime}\right) .
$$

In other words, $\left(E_{A}(\phi), \tilde{\nu}\right)$ is an $L$-gerbe in $\mathcal{F}^{\prime}$.

Suppose that $f:(\phi, \nu) \rightarrow\left(\phi^{\prime}, \nu^{\prime}\right)$ is a morphism of the 2-cocycle category $h\left(*, p_{\mathcal{F}} / L\right)$. Then there is a full subpresheaf $\mathcal{F}^{\prime \prime} \subset \operatorname{Iso}(\operatorname{Gr}(\mathcal{C}))$ containing $\mathcal{F}$, such that $\mathcal{F} \subset \mathcal{F}^{\prime \prime}$ is a weak equivalence and such that the associated gerbes $E_{A}(\phi)$ and $E_{A^{\prime}}\left(\phi^{\prime}\right)$ are gerbes in $\mathcal{F}^{\prime \prime}$. There is a diagram of homotopies

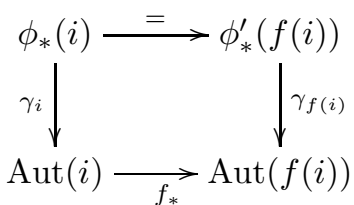

where $\operatorname{Aut}(i)$ is the sheaf of automorphisms of $i$ in $E_{A}(\phi)$ and $\operatorname{Aut}(f(i))$ is the sheaf of automorphisms of $f(i)$ in $E_{A^{\prime}}\left(\phi^{\prime}\right)$. Then the morphisms $\nu_{i}: \phi(i) \rightarrow L$ and $\nu_{f(i)}^{\prime}: \phi^{\prime}(f(i)) \rightarrow L$ coincide on $\phi(i)=\phi^{\prime}(f(i))$ since $f$ is a morphism of the cocycle category $h\left(*, B\left(p_{\mathcal{F}} / L\right)\right)$. Furthermore, the vertical isomorphisms uniquely determine the natural isomorphisms $\tilde{\nu}: \operatorname{Aut}(i) \rightarrow L$ and $\tilde{\nu}^{\prime} f_{*}: \operatorname{Aut}(f(i)) \rightarrow L$, respectively. It follows that 
the map $f_{*}: E_{A}(\phi) \rightarrow E_{A^{\prime}}\left(\phi^{\prime}\right)$ defines a morphism of $L$-gerbes in $\mathcal{F}^{\prime \prime}$. We therefore have a well defined function

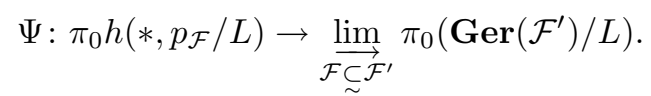

Theorem 27. Suppose that $\mathcal{F}$ is a full subpresheaf of $\mathbf{I s o}(\operatorname{Gr}(\mathcal{C}))$ and that $L \in \operatorname{St}(\pi \mathcal{F})$ is a band. Then the function $\Psi$ is a bijection.

Proof: Suppose that $\mathcal{F} \subset \mathcal{F}^{\prime \prime}$ is a weak equivalence of full subpresheaves of $\operatorname{Iso}(\operatorname{Gr}(\mathcal{C}))$. Then the diagram

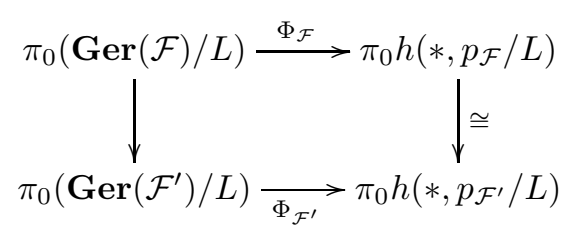

commutes, where the indicated vertical map is a bijection since the comparison map $\left.B\left(p_{\mathcal{F}} / L\right)\right) \rightarrow B\left(p_{\mathcal{F}^{\prime}} / L\right)$ is a local weak equivalence. It follows that the maps $\Phi_{\mathcal{F}^{\prime}}$ together induce a function

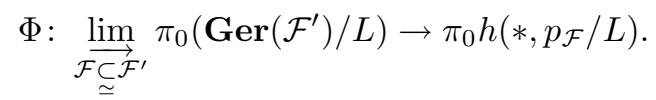

The function $\Phi$ is the inverse of $\Psi$.

Corollary 28. Suppose that $\mathcal{F}$ is a full subpresheaf of $\operatorname{Iso}(\operatorname{Gr}(\mathcal{C}))$ and that $L \in \operatorname{St}(\pi \mathcal{F})$ is a band. Then there are bijections

$$
\left[*, B\left(p_{\mathcal{F}} / L\right)\right] \cong \pi_{0} h\left(*, p_{\mathcal{F}} / L\right) \cong{\underset{\mathcal{F}}{\mathcal{F} \subseteq \mathcal{F}^{\prime}}}_{\lim _{0}} \pi_{0}\left(\operatorname{Ger}\left(\mathcal{F}^{\prime}\right) / L\right) .
$$

\section{References}

[1] L. Breen, Bitorseurs et cohomologie non abélienne, in: "The Grothendieck Festschrift", Vol. I, Progr. Math. 86, Birkhäuser Boston, Boston, MA, 1990, pp. 401-476.

[2] J. Giraud, "Cohomologie non abélienne", Die Grundlehren der mathematischen Wissenschaften 179, Springer-Verlag, Berlin-New York, 1971.

[3] P. G. Goerss And J. F. Jardine, "Simplicial homotopy theory", Progress in Mathematics 174, Birkhäuser Verlag, Basel, 1999.

[4] S. Hollander, A homotopy theory for stacks, Israel J. Math. 163 (2008), 93-124. 
[5] J. F. Jardine, Simplicial presheaves, J. Pure Appl. Algebra 47(1) (1987), 35-87.

[6] J. F. JARDine, Stacks and the homotopy theory of simplicial sheaves. Equivariant stable homotopy theory and related areas (Stanford, CA, 2000), Homology Homotopy Appl. 3(2) (2001), 361-384 (electronic).

[7] J. F. JARdine, Cocycle categories, Preprint, to appear in Abel Symposium Proceedings (2006), arXiv:math/0605198v1.

[8] J. F. JARdine, Diagrams and torsors, K-Theory 37(3) (2006), 291-309.

[9] J. F. JARDine, Fibred sites and stack cohomology, Math. Z. 254(4) (2006), 811-836.

[10] J. F. JARDine, Torsors and stacks, Mediterr. J. Math. 3(2) (2006), $251-258$.

[11] J. F. JARdine, Fields Lectures: Simplicial presheaves (2007), at http: //www.math. uwo.ca/ jardine/papers/Fields-01.pdf.

[12] A. JoyAl And M. TiERnEy, Strong stacks and classifying spaces, in: "Category theory" (Como, 1990), Lecture Notes in Math. 1488, Springer, Berlin, 1991, pp. 213-236.

[13] G. Laumon And L. Moret-Bailly, "Champs algébriques", Ergebnisse der Mathematik und ihrer Grenzgebiete. 3. Folge. A Series of Modern Surveys in Mathematics [Results in Mathematics and Related Areas. 3rd Series. A Series of Modern Surveys in Mathematics] 39, Springer-Verlag, Berlin, 2000.

[14] Z. Luo, Closed model categories for presheaves of simplicial groupoids and presheaves of 2-groupoids, Preprint (2003), arXiv:math/0301045v1.

[15] I. MoERDiJK, Bisimplicial sets and the group-completion theorem, in: "Algebraic K-theory: connections with geometry and topology" (Lake Louise, AB, 1987), NATO Adv. Sci. Inst. Ser. C Math. Phys. Sci. 279, Kluwer Acad. Publ., Dordrecht, 1989, pp. 225-240.

Mathematics Department

University of Western Ontario

London, Ontario N6A 5B7

Canada

E-mail address: jardine@uwo.ca

Primera versió rebuda el 4 de setembre de 2008, darrera versió rebuda el 29 de juny de 2009. 